\title{
Perfil dermatoglífico e bullying escolar em crianças e adolescentes
}

\author{
School profile dermatoglyphic and bullying in teens
}

\author{
Marcela Almeida Zequinão, ${ }^{1}$ Pâmella de Medeiros, ${ }^{2}$ Paola Cidade Cordeiro, ${ }^{2}$ \\ Rudy Nodari Junior, 3 Fernando Luiz Cardoso 2 \\ "Universidade do Minho, Largo do Paço, Braga, Portugal. \\ ZUniversidade do Estado de Santa Catarina (CEFID/UDESC), Florianópolis, SC, Brasil. \\ Universidade do Oeste de Santa Catarina (UNOEST), Joaçaba, SC, Brasil.
}

Recebido em: 13/02/2017 / Aceito em: 09/06/2017 / Publicado em: 30/06/2017

pamellademedeiros@hotmail.com

\section{RESUMO}

O fenômeno bullying vem sendo tema de diversas pesquisas nos últimos 30 anos, contudo, apenas recentemente, com o intuito de tentar entender e explicar o envolvimento de crianças e adolescentes nesse comportamento violento, pesquisadores têm apontado características individuais como possíveis indicadores de envolvimento nos papéis de participação do bullying escolar. Objetivo: analisar as relações entre aspectos genéticos e participação no bullying escolar, por meio do perfil dermatoglífico de crianças e adolescentes de escolas de Florianópolis, SC. Material e Método: participaram 409 crianças e adolescentes do $3^{\circ}-7^{\circ}$ ano do Ensino Fundamental, em duas escolas públicas. Para avaliação da marca genética utilizou-se o Leitor Dermatoglífico e para os papéis no bullying, utilizou-se uma Escala Sócio Métrica. Resultados: uma associação negativa entre ser vítima e prevalência do desenho LU foi encontrada, bem como uma associação positiva entre ser agressor e prevalência do desenho LR. Conclusão: as diferenças entre os desenhos LR e LU vêm sendo estudadas recentemente e ainda não apontam resultados conclusivos sobre as características específicas de indivíduos com esses tipos de desenhos e possíveis diferenças entre eles. Mais estudos são necessários para investigar se existem relações entre esses desenhos e questões comportamentais e de personalidade, podendo influenciar a participação no bullying.

Palavras-chave: Dermatoglifia; Genética; Intimidação Escolar

\section{ABSTRACT}

The bullying phenomenon has been subject of several studies over the past 30 years, however, only recently, while aiming to understand and explain the involvement of children and adolescents in this violent behavior, researchers have identified individual characteristics as possible indicators of involvement in the participation roles in school bullying. Objective: to analyse the relationships between genetic aspects and participation in school bullying, using the dermatoglyphic profile of children and teenagers from schools of Florianópolis, SC. Materials and Method: 409 children and adolescents participated in the 3rd-7th year of the elementary school in two public schools. To assess the genetic fingerprint Reader Dermatoglyphic was used and for the roles in the bullying, we used a Sociometric Scale. Results: a negative association between being a victim and prevalence of drawing $L U$ was found, as well as a positive association between aggressor and prevalence of $L R$ design. Conclusions: the differences between the drawings $L R$ and $L U$ have been studied recently, and still not point conclusive results about the specific features of individuals with these types of designs and possible differences between them. Further studies are required to investigate if there are relationships between these drawings and behavioural issues and personality, and may influence participation in bullying. School 


\section{INTRODUÇÃO}

A disputa de poder entre iguais pode gerar um fenômeno denominado na literatura internacional de bullying, que é considerado um conjunto de atitudes agressivas, intencionais e repetitivas, que ocorrem sem motivação evidente, adotado por um ou mais alunos contra um ou outros, causando dor, angústia e terrível sofrimento às vítimas. ${ }^{1-7}$ Esses atos violentos acontecem sistematicamente e intencionalmente; todavia, ocorrem de forma sigilosa e despercebida, não manifestando ações explícitas que possam ser identificadas por terceiros, fazendo com que a vítima não tenha possibilidade de defesa. ${ }^{8-11}$ É também uma estratégia coerciva que visa alcançar e manter posições mais elevadas na hierarquia social do grupo. ${ }^{12,13}$

A ocorrência deste fenômeno é considerada bas tante comum entre crianças e adolescentes. Estima-se que entre $10 \%$ a $30 \%$ das crianças em idade escolar de todo o mundo estejam envolvidos em alguma situação de bullying. ${ }^{14,15}$ Autores também acreditam que essas porcentagens podem variar de acordo com o nível socioeconômico dos países, sendo que em países de alta renda, a estimativa aproxima-se de $5 \%$ a $30 \%,{ }^{16}$ e nos de média e baixa renda entre $10 \%$ a $60 \% .{ }^{17}$

Embora o fenômeno bullying venha sendo tema de diversas pesquisas nos últimos 30 anos, apenas recentemente, com o intuito de tentar entender e explicar o envolvimento de crianças e adolescentes nesse comportamento violento, pesquisadores têm apontado características individuais como possíveis indicadores de envolvimento nos papéis de participação do bullying escolar. ${ }^{18}$

Características preexistentes vêm sendo indicadores, a nível individual, para a participação no bullying escolar, podendo aumentar a probabilidade de se tornarem agressores ou alvos de intimidação. ${ }^{19,20}$ Dentre esses indicadores, pode-se citar sexo, temperamento, agressividade, competitividade por domínio social e internalização e externalização de sintomas, como sendo aspectos parcialmente influenciados por fatores genéticos e que são extremamente importantes de serem considerados na tentativa de compreender e combater estes comportamentos. ${ }^{19-23}$

Estudos indicam que o ambiente é particularmente crucial na vitimização em idades mais jovens; no entanto, com o passar dos anos, a influência das características genéticas torna-se cada vez mais importante, ${ }^{19}$ ao ponto que no estudo de Ball, Taylor e Caspi ${ }^{24} \mathrm{com}$ gêmeos de 10 anos de idade, os marcadores genéticos explicaram mais de dois terços das diferenças individuais em vitimização infantil nessa idade. Outros estudos confirmam estes achados e também indicam que, além da ocorrência de vitimização, a persistência desse papel, bem como questões relacionadas à externalização da agressividade, está intimamente relacionada a fatores genéticos. ${ }^{19,20,22,25,26}$

Marcadores genéticos são características únicas de cada pessoa, desenvolvidas durante a fase pré-natal e que se mantém, durante toda a vida do indivíduo. Dentre os marcadores genéticos mais conhecidos estão às impressões digitais. Compreende-se impressões digitais por representações dérmicas das características genéticas, ${ }^{22,27}$ que se formam no ser humano no estado intra-uterino, a partir de estrato blastogênico entre o $3^{\circ}$ e o $6^{\circ}$ mês de gestação e não se alteram durante toda a vida, fazendo com que o envolvimento pós-natal não tenha qualquer papel na variabilidade dermatoglífica, exceto em algumas condições patológicas. ${ }^{28-30}$ As impressões digitais são marcas genéticas universais que possuem significativas características populacionais e étnicas. ${ }^{31}$

$O$ estudo das impressões digitais deu origem ao termo dermatoglifia, proposto por Cummins e Midlo ${ }^{27}$ introduzido em 1926, na $42^{\text {a }}$ Sessão Anual da Associação Americana de Anátomos e recebeu a classificação de método no ramo da Ciência Médica do estudo do relevo. ${ }^{32}$ A etimologia da palavra veio do latim, dermo, significa "pele", e do grego, glypha, "gravar" e assim definiu-se o nome da ciência que estuda o relevo das cristas cutâneas e desenhos das pontas dos dedos, palmas das mãos e das plantas dos pés. ${ }^{29,30,33}$

A dermatoglifia tornou-se um instrumento que utiliza as impressões digitais como marcadores genéticos para serem estudadas em associação com as qualidades básicas do indivíduo, suas potencialidades genéticas, bem como a tipologia de suas fibras. ${ }^{22,34-40}$ Com base nessas informações, o presente estudo tem como objetivo analisar as relações entre a participação no bullying escolar e aspectos genéticos, por meio do perfil dermatoglífico de crianças e adolescentes

\section{MÉTODO}

\section{Caracterização da pesquisa}

Um estudo transversal de cunho exploratório e amostragem intencional foi realizado na região metropolitana de Florianópolis, entre fevereiro e dezembro de 2012. Todos os participantes estiveram presentes, individualmente, nas duas etapas da coleta de dados. Na primeira etapa, os participantes responderam uma Escala Sócio métrica sobre a participação dos colegas no bullying, enquanto a segunda etapa consistiu na coleta da impressão digital. Ambos os instrumentos foram aplicados e supervisionados por dois pesquisadores treinados para esclarecer qualquer dúvida dos participantes. Esta pesquisa faz parte de um macroprojeto aprovado junto ao Comitê de Ética em Pesquisas com Seres Humanos sob processo 5439/2011, $\mathrm{n}^{\circ}$ expediente 75/2011.

\section{Participantes}

Os participantes deste estudo foram crianças e adolescentes do $3^{\circ}$ ao $7^{\circ}$ ano do Ensino Fundamental, de ambos os sexos, com idades entre 8 e 16 anos, matriculadas no período vigente do estudo em duas escolas públicas municipais. De acordo com os objetivos do macroprojeto, ao qual este estudo faz parte, um tamanho de amostra foi calculado assumindo um alfa de 0,05 e poder de $50 \%$. A partir do cálculo amostral, o mínimo esperado eram 316 participantes. Tendo em vista que todas as crianças do $3^{\circ}$ ao $7^{\circ}$ ano foram convidadas a participar, o tamanho da amostra final foi de 409 participantes. A seleção das escolas participantes foi intencional, pois se deu pela indicação da secretária de educação do município, nas quais as escolas selecionadas eram as que mais se enquadravam no perfil proposto pelo estudo. Após a seleção das escolas, todos os alunos do $3^{\circ}$ ao $7^{\circ}$ ano foram convidados a participar, no entanto, apenas os que entregaram o 
termo de consentimento assinado pelos pais foram selecionados. O único critério de exclusão adotado no recrutamento dos participantes foi apresentar alguma deficiência intelectual, que impedisse a compreensão dos instrumentos, com base na indicação da Associação Escolar Especial das escolas.

\section{Instrumentos}

A fim de caracterizar os participantes no início do estudo, variáveis sócio demográficas foram obtidas. As variáveis sócio demográficas foram: idade, número de pessoas em casa, número de irmãos, ordem do nascimento, número de escolas que já estudou, número de casas que já morou e cor/raça.

Para a avaliação da marca genética foi utilizado o protocolo sugerido por Cummis e Midlo, ${ }^{27}$ por meio do Leitor Dermatoglífico, ${ }^{41}$ que consiste no processamento e análise de impressões digitais pelo Método Dermatoglífico. Utilizou-se um processo informatizado para leitura dermatoglífica, por meio do Leitor Dermatoglífico constituído de um scanner óptico de rolamento, que coleta, interpreta a imagem e constrói, em código binário, um desenho, que é capturado por software específico de tratamento e reconstrução de imagens reais e binarizadas em preto e branco. Com o Leitor Dermatoglífico, a partir da imagem coletada, o avaliador realiza a marcação dos pontos núcleo e delta dos desenhos das impressões digitais dos 10 dedos das mãos de cada criança. Então, o software faz a identificação qualitativa da imagem e quantitativa de linhas, gerando uma planilha informatizada com o tipo de desenho e o número total de linhas. ${ }^{41}$ As variáveis analisadas neste instrumento foram: somatório total de linhas das mãos esquerda (SQTLE) e direita (SQTLD); somatório total de linhas de ambas as mãos (SQTL); frequência dos desenhos Arco (A), Presilha Radial (LR) e Ulnar (LU) e Verticilo (W); e número de deltas de todos os dedos das mãos (D10).

Para descrever os possíveis papéis de participação no bullying, utilizou-se a Escala Sócio Métrica constituída de informações baseadas em diversos instrumentos, ${ }^{42-44}$ e que foram adaptadas, ${ }^{45}$ sendo essa última versão, a utilizada no estudo. Esse exame consiste em diversas perguntas do cotidiano da sala de aula que envolve comportamentos característicos de vítimas e agressores de bullying escolar. Cada participante citou o nome de três colegas de classe que mais estavam envolvidos nessas situações. Assim, todos os alunos tiveram dois escores gerados pelo número de vezes em que foram citados nas questões sobre ser vítima e agressor no ambiente escolar. Em relação à confiabilidade interna do instrumento, a Escala Sócio Métrica apresentou um bom nível de consistência interna, com um alfa de Cronbach igual $\mathrm{a}=0.800$.

\section{Análises dos dados}

Utilizou-se o teste de normalidade de Kolmogorov Smirnov para verificar se os dados atendiam os pressupostos paramétricos. Como a distribuição dos dados não foi normal, realizou-se em seguida a estatística descritiva e estatística inferencial por meio dos testes Mann-Whitney, para comparação entre grupos, e correlação de Spearman, para verificar a relação entre as variáveis.

Os dados da pesquisa foram tabulados e analisados no programa computadorizado Statistical Package for the Social Science (SPSS for Windows), versão 20.0 e para todos os testes foi adotado um intervalo de confiança de $95 \%(p<0,05)$.

\section{RESULTADOS}

Primeiramente foram obtidos os dados sócio demográficos dos participantes para melhor caracterizá-los. Percebeu-se que os mesmos formam um grupo bastante homogêneo, no qual os participantes tiveram média de idade de, aproximadamente 11 anos, sendo a maioria da cor branca (Tabela 1).

Quando analisado o perfil dermatoglífico dos participantes, identificou-se que a única diferença encontrada entre os sexos foi em relação à prevalência do desenho LR, na qual verificou-se que os meninos apresentaram mais este tipo de desenho, quando comparados às meninas $(M=, 53$ e $\mathrm{sd}=, 731$ e $\mathrm{M}=, 36 \mathrm{e}$ $\mathrm{sd}=, 633$, respectivamente) (Tabela 2).

No entanto, quando comparados os perfis dermatoglíficos com os possíveis papéis de participação no bullying escolar, verificou-se uma associação negativa entre ser vítima e a prevalência do desenho LU, bem como foi encontrada uma associação positiva entre ser agressor e a prevalência do desenho LR (Figura 1).

Tabela 1 - Perfil sócio demográfico dos participantes.

\begin{tabular}{|c|c|c|c|c|c|c|}
\hline & \multicolumn{2}{|c|}{ MENINOS ( $=207)$} & \multicolumn{2}{|c|}{ MENINAS $(\mathrm{N}=202)$} & \multirow[b]{2}{*}{$\mathbf{U}$} & \multirow[b]{2}{*}{$\mathbf{P}$} \\
\hline & $\mathrm{x}$ & Sd & $\mathrm{X}$ & Sd & & \\
\hline Idade & 11,14 & 1,61 & 110,90 & 1,82 & 18825,00 & 0,910 \\
\hline $\mathrm{N}^{\circ}$ de pessoas em casa & 4,75 & 1,79 & 4,77 & 1,74 & 20422,50 & 0,680 \\
\hline $\mathrm{N}^{\circ}$ de irmãos & 2,79 & 2,22 & 2,61 & 1,97 & 20023,50 & 0,450 \\
\hline Ordem do nascimento & 2,73 & 2,00 & 2,60 & 1,81 & 20204,50 & 0,540 \\
\hline $\mathrm{N}^{\circ}$ de escolas que já estudou & 2,50 & 1,14 & 2,45 & ,962 & 20788,00 & 0,990 \\
\hline $\mathrm{N}^{\circ}$ de casas que já morou & 1,78 & 2,66 & 1,79 & 1,88 & 20064,00 & 0,520 \\
\hline Cor ou raça & $\mathbf{N}$ & $\%$ & $\mathbf{N}$ & $\%$ & $\chi^{2}$ & $\mathbf{P}$ \\
\hline Preta & 20 & 9,7 & 18 & 8,9 & & \\
\hline Branca & 142 & 68,6 & 148 & 73,3 & & \\
\hline Parda & 12 & 5,8 & 4 & 2,0 & 4,184 & 0,242 \\
\hline Morena & 33 & 15,9 & 32 & 15,8 & & \\
\hline Total & 193 & $100 \%$ & 195 & $100 \%$ & & \\
\hline
\end{tabular}

*X: média; sd: Desvio padrão; p: nível de significância $\leq 0.05$; U: teste de Mann Whitney; $x^{2}$ Teste do qui quadrado. 
Tabela 2 - Comparação entre os sexos em relação ao perfil dermatoglífico de crianças e adolescentes, em situação de alta vulnerabilidade social.

\begin{tabular}{lcccccc}
\hline VARIAVEL & \multicolumn{7}{c}{ MENINOS $\mathbf{( N = 2 0 7 )}$} & \multicolumn{2}{c}{ MENINAS $(\mathbf{N}=\mathbf{2 0 2})$} & $\mathbf{U}$ & $\mathbf{P}$ \\
\hline SOTLE & $\mathbf{X}$ & $\mathbf{S d}$ & $\mathbf{X}$ & $\mathbf{S d}$ & 13016,00 & 0,187 \\
SOTLD & 55,53 & 21,02 & 52,08 & 22,73 & 13399,50 & 0,374 \\
SOTL & 56,40 & 18,72 & 53,98 & 20,80 & 13262,00 & 0,297 \\
A & 111,93 & 38,42 & 106,02 & 42,57 & 13684,50 & 0,425 \\
LR &, 44 & 1,15 &, 71 & 1,83 & 12410,50 & 0,017 \\
LU &, 53 &, 731 &, 36 &, 633 & 13656,50 & 0,543 \\
W & 6,39 & 2,38 & 6,49 & 2,56 & 13670,50 & 0,551 \\
D10 & 2,64 & 2,63 & 2,44 & 2,49 & 13288,00 & 0,307 \\
\hline
\end{tabular}

*Md: Mediana, IQ: Interquartil; U: Teste de Mann Whitney; $p$ : nível de significância; $p \leq 0.05$

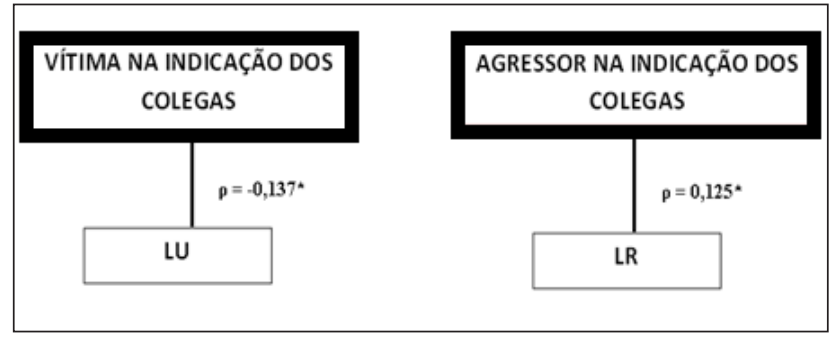

*LU: Presilha Ulnar; LR: Presilha Radial; p: nível de significância $p \leq 0.05$ Fonte: produção dos próprios autores.

Figura 1 - Relação entre os possíveis papéis de participação no bullying escolar, segundo a percepção dos colegas, em relação ao perfil dermatoglífico de crianças e adolescentes, em situação de alta vulnerabilidade social.

\section{DISCUSSÃO}

O objetivo deste estudo foi analisar as relações entre a participação no bullying escolar e aspectos genéticos, por meio do perfil dermatoglífico de crianças e adolescentes. Verificou-se que poucas variáveis do perfil darmatoglífico diferenciaram-se entre os participantes deste estudo. Os principais resultados encontrados foram em relação ao tipo de desenho Presilha. Encontrou-se diferença entre o número de LR e os sexos, nos quais meninos apresentaram mais esse tipo de desenho, quando comparados às meninas. Bem como, as relações encontradas com os papéis de participação no bullying escolar, também fizeram referência ao desenho Presilha, sendo que as vítimas apresentaram correlação negativa com LU, enquanto os agressores apresentaram correlação positiva com LR.

No entanto, esses resultados são pouco esclarecedores, tendo em vista que as diferenças entre os desenhos LR e LU vêm sendo estudadas recentemente, e ainda não apontam resultados conclusivos sobre as características específicas de indivíduos, com esses tipos de desenhos e as possíveis diferenças entre eles. 0 que se sabe, em relação ao desenho "L", está mais voltado para aspectos das capacidades físicas e motoras do indivíduo, como por exemplo, que a presença de " $L$ " está associada à velocidade e a força explosiva com os baixos níveis de resistência ${ }^{46-48}$. Ao passo que, quando encontramos indivíduos com predominância desse tipo de desenho, classifica-se como sendo anaeróbio, tendo uma predominância de fibras de contração rápida ${ }^{49}$ e a uma predisposição à maturação tardia. ${ }^{50}$

Posto que estudos venham apontando fatores genéticos, como sendo marcadores importantes para a compreensão do fenômeno bullying, alguns dados indicam que, embora características individuais, como a agressividade, sejam preditivas de violência escolar, o que se verifica é que, em contextos de aceitação e reforço positivo desse comportamento, a associação torna-se muito mais forte, ${ }^{20}$ indicando que fatores ambientais podem ser tão importantes quanto fatores genéticos.

Neste estudo, encontram-se poucas variáveis do perfil darmatoglífico que se diferenciaram entre os participantes. Acredita-se que, no grupo estudado, os fatores ambientais foram os principais responsáveis pelo envolvimento nesse tipo de comportamento. Contudo, mais estudos na área, são necessários para investigar as características dos desenhos LR e LU, para que então, seja possível verificar se esse marcador genético tem relação com questões comportamentais e de personalidade, podendo influenciar nos comportamentos de crianças e adolescentes, com envolvimento no bullying escolar.

\section{AGRADECIMENTOS}

Agradecemos o apoio pela Coordenação de Aperfeiçoamento de Pessoal de Nível Superior - CAPES sob processo $n^{\circ} 0815 / 14-4$.

\section{REFERÊNCIAS}

1. Olweus D. Bullying at school: basic facts and effects of a school based intervention program. J Child Psychol Psychiatry 1994;35(7):1171-90. ISSN 0021-9630. doi: 10.1111/j.14697610.1994.tb01229.x Disponível em: < http://www.ncbi. nlm.nih.gov/pubmed/7806605 >.

2. Lopes Neto, A. Bullying: comportamento agressivo entre estudantes. J Pediatr (Rio J) 2005;81(5):164-72. doi: 0021-7557/05/81-05-Supl/S16

3. Pereira B. Para uma escola sem violência: estudo e prevenção das práticas agressivas entre crianças. 2006. Lisboa: Fundação Calouste Gulbekian.

4. Melim F, Pereira B. Prática desportiva, um meio de prevenção do bullying na escola? Movimento 2013;19(2):55-77.

5. Rocha M, Costa, CDA, Passos Neto IDF. Bullying e o papel da sociedade. Cadernos de Graduação - Ciências Humanas e Sociais 2013;1(16):191-9. 
6. Medeiros P, Zequinão MA.; Cardoso FL. Bullying e a relação entre atividade física e coordenação motora: uma revisão sistemática. Cinergis 2014;15(3):158-62. doi: 10.17058/ cinergis.v15i3.5075

7. De Oliveira WA, Silva MAI, Mello FCM, Porto DL,Yoshinaga ACM.; The causes of bullying: results from the National Survey of School Health (PeNSE). Rev Lat Am Enfermagem 2015;23(2):275-82. ISSN 1518-8345. Disponível em: < http://www.ncbi.nlm.nih.gov/pubmed/26039298 >. doi: 10.1590/0104-1169.0022.2552

8. Rigby K. A meta-evaluation of methods and approaches to reducing bullying in pre-schools andin early primary school in Australia. 2002. Canberra: Commonwealth AttorneyGeneral's Department.

9. Smith P. Bullying: Recent Developments. Child and Adolescent Mental Health 2004;9(3):98-103. doi: 10.1111/j.1475-3588.2004.00089.x

10. Nogueira RDPDA. Violência nas escolas e juventude: um estudo sobre o bullying escolar [Dissertação]. São Paulo: PUC-SP; 2007.

11. Raimundo $\mathrm{R}$, Seixas $\mathrm{S}$. Comportamentos de bullying no $1^{\circ}$ ciclo: estudo de caso numa escola de Lisboa. Interacções 2009;13:164-86.

12. Pronk J, Frits AG, Tjeert $O$, Langha DM, Willemen AM. Children's intervention strategies in situations of victimization by bullying: social cognitions of outsiders versus defenders. J Sch Psychol 2013;51(6):669-82. ISSN 1873-3506. doi: 10.1016/j.jsp.2013.09.002 Disponível em: < http://www. ncbi.nlm.nih.gov/pubmed/24295142 >

13. Sekol, I. Peer violence in adolescent residential care: A qualitative examination of contextual and peer factors. Children and Youth Services Review 2013;35;1901-12. doi: 10.1016/j.childyouth.2013.09.006

14. Molina BL, Williamson AA, Pulido R, Calderón S. Adaptation of the Participant Role Scale (prs) in a spanish youth sample: measurement invariance across gender and relationship with sociometric status. Journal of Interpersonal Violence 2014;29(16):2904-30. doi: 10.1177/0886260514527822

15. Rettew DC, Pawlowski S. Bullying. Child Adolesc Psychiatr Clin N Am 2016;25(2):235-42. ISSN 1558-0490. doi: 10.1016/j.chc.2015.12.002 Disponível em: < http://www. ncbi.nlm.nih.gov/pubmed/26980126 >

16. Currie C, Zanotti C, Morgan A, Currie D, Roberts MLC, Samdal O, Smith ORF, Barnekow V. Social determinants of health and wellbeing among young people. Health Behaviour in School-aged Children (HBSC) study: International report from the 2009/2010 survey health policy for children and adolescents. Copenhagen: WHO Regional Office for Europe. 2010.

17. Fleming $\mathrm{LC}$, Jacobsen $\mathrm{KH}$. Bullying among middle-school students in low and middle income countries. Health Promot Int 2010;25(1):73-84. ISSN 1460-2245. Disponível em: < http://www.ncbi.nlm.nih.gov/pubmed/19884243 >. doi: 10.1093/heapro/dap046

18. Cook C, Williams KR, Guerra NG, Kim T, Sadek S. Predictors of Bullying and Victimization in Childhood and Adolescence: A Meta-analytic Investigation. School Psychology Quarterly 2010;25(2):65-83. doi: 10.1037/a0020149

19. Bowes L, Maughan B, Maughan B, Ball H, Shakoor S, Ouellet-morin I, Caspi A, Moffitt TE, Arseneault L. Chronic bullying victimization across school transitions: the role of genetic and environmental influences. Dev Psychopathol 2013;25(2):333-46. doi: 10.1017/S0954579412001095

20. Nocentini A, Menesini E, Salmivalli C. Level and change of bullying behavior during high school: a multilevel growth curve analysis. J Adolesc 2013;36(3):495-505. doi: 10.1016/j.adolescence.2013.02.004

21. Abramovay M, Castro MG, Pinheiro LC, Lima F, Martinelli C. Juventude, Violência e Vulnerabilidade Social na América Latina: Desafios para Políticas Públicas. Brasília: Unesco Brasil; 2002.

22. Abramova T, Nikitina, TM, Izaak, SI, Kochetkova NI. [Asymmetry of signs of finger dermatoglyphics, physical potential and physical qualities of a man]. Morfologiia 2000; 118(5):56-9. ISSN 1026-3543. Disponível em: < http:// www.ncbi.nlm.nih.gov/entrez/query.fcgi?cmd $=$ Retrieve \&db $=$ PubMed\&dopt $=$ Citation\&list $\_$uids $=11452431>$.

23. Assis $S$, Pesce R, Avanci J. Resiliência: enfatizando a proteção dos adolescentes. Porto Alegre: ARTMED; 2006.

24. Ball HA, Arseneault L, Taylor A, Maughan B, Caspi A, Moffitt TE. Genetic and environmental influences on victims, bullies and bully-victims in childhood. J Child Psychol Psychiatry 2008;49(1):104-12. doi: 10.1111/j.1469-7610.2007.01821.x

25. Brendgen M, Boivin M, Vitaro F, Girard A, Dionne G, Pérusse D. Gene-environment interaction between peer victimization and child aggression. Dev Psychopathol 2008;20(2):455-71. doi: 10.1017/S0954579408000229.

26. Menesini E, Modena M, Tani F. Bullying and victimization in adolescence: concurrent and stable roles and psychological health symptoms. J Genet Psychol 2009;170(2):115-33. doi: 10.3200/GNTP.170.2.115-134

27. Cummins $H$, Midlo C. Finger Prints, Palmas, and Soles: An Introduction to Dermatoglyphics. New York: Dover Publication. 1961

28. Nikitiuk B, Filipov V. Complexo Dermatoglífico que acompanham o alto desenvolvimento das qualidades motoras: Método biológico de gêmeos, médico e esporte. 1984.

29. Fernandes Filho, J. Impressões Dermatoglíficas - Marcas Genéticas na Seleção dos tipos de esporte e lutas (a exemplo de Desportista do Brasil). 1997. Moscou.

30. Assef M, Oliveira A, Alonso L. Dermatoglifos como preditores da coordenação motora em atletas da seleção brasileira feminina de futebol sub-17. Efdeportes.com, Revista Digital 2009;14(132).

31. Castanhede A, Dantas P, Fernandes Filho, J. Perfil dermatoglífico e somatotípico de atletas de Futebol de Campo masculino, de alto rendimento no Rio de Janeiro - Brasil. Fitness \&amp; Performance Journal 2003;2(4):234-39. doi:10.3900/ fpj.2.4.234.p

32. Fazolo E, Cardoso PG, Tuche W, Menezes IC, Teixeira ME, Portal MND, Nunes RMA, Costa G, Dantas PMS, Fernandes Filho $\mathrm{J}$. A dermatoglifia e a somatotipologia no alto rendimento do beach soccer - Seleção Brasileira. Revista de Educação Física 2005;130:45-51.

33. Matsuyama N, Ito $\mathrm{Y}$. The frequency of fingerprint type in parents of children with Trisomy 21 in Japan. J Physiol Anthropol 2006;25(1):15-21. ISSN 1880-6791. doi: 10.2114/jpa2.25.15 Disponível em: < http://www.ncbi.nlm. nih.gov/entrez/query.fcgi?cmd $=$ Retrieve\&db $=$ PubMed\&dopt $=$ Citation\&list_uids $=16617204>$.

34. Beiguelman B. Dinâmica dos genes nas famílias e nas populações. Revista Brasileira de Genética 1995;(2).

35. Łopuszańska M, Jankowska EA. [Dermatoglyphic morphology in some diseases]. Pol Merkur Lekarski 2001;11(63):282-6. ISSN 1426-9686. Disponível em: < http://www.ncbi.nlm. nih.gov/pubmed/11761831 >.

36. Dantas P, Fernandes Filho J. Identificação dos perfis, 
genético, de aptidão física e somatípico que caracterizam atletas masculinos, de alto rendimento, participantes do futsal adulto, no Brasil. Fitness e Performance Journal 2002;1(1):28-36. doi:10.3900/fpj.1.1.28.p

37. Dantas $\mathrm{P}$, Alonso L, Fernandes Fllho J. A dermatoglifia no futsal brasileiro de alto rendimento. Fitness e performance Journal 2004;3(3):136-42. doi: 5133doi:10.3900/fpj.3.3.136.p

38. Fernandes $P$, Fernandes Filho J. Dermatoglifia, somatotipia e consumo máximo de oxigênio em jogadores de futebol de campo, portadores e não portadores de paralisia cerebral. In: (Ed.). Atividade física e ciências da saúde.Atividade física e ciências da saúde. Rio de Janeiro: Shape 2005;109-123. doi:10.3900/fpj.3.3.157.

39. Fernandes Filho J, Dantas P, Fernandes P. Genética e treinamento esportivo: o uso prático da dermatoglifia. In: DACOSTA, L. (Ed.). Atlas do Esporte no Brasil. 2006. Rio de Janeiro: CONFEF

40. Linhares M, Ferreira DDC, Fernandes Filho J. Avaliação do perfil pessoal de adolescentes talentosos utilizando suas características dermatoglíficas. Revista Meta: Avaliação 2009;1(2):220-236. doi: 10.22347/2175-2753v1i2.43

41. Nodari Junior R, Heberle A, Emygdio RE, Knackfuss MI. Impressões Digitais para Diagnóstico em Saúde: Validação de Protótipo de escaneamento Informatizado. Revista de Saúde Pública 2008;10(5):767-776.

42. Pereira BO, Mendonça D, Neto C, Velente L, Smith PK. Bullying in portuguese schools. School Psychology International 2004;25(2):207-22.
43. Smith P. Bullying: Recent Developments. Child and Adolescent Mental Health 2004;9(3):98-103. doi: 10.1111/j.1475-3588.2004.00089.x

44. Freire $I$, Simão $A$, Ferreira $A$. O estudo da violência entre pares no $3^{\circ}$ ciclo do ensino básico: um questionário aferido para a população escolar portuguesa. Revista Portuguesa de Educação 2006;19(2):157-83.

45. Nogueira RDPDA. Violência nas escolas e juventude: um estudo sobre o bullying escolar [Dissertação]. São Paulo: PUC-SP; 2007.

46. Abramova T, Nikitina T, Ozolin N. Impressões dermatoglíficas: marcas genéticas nopotencial energético do homem. Congresso Científico de Moscou; 1995. Moscou: 2005.

47. Medeiros H, Rocha V, Fernandes Filho J. Dermatoglifia e maturação. Atividade física e ciências da saúde. Rio de Janeiro: Shape; 2005.

48. Cunha Júnior AT, Cunha ACPT, Scheneider AT, Dantas PMS. Características dermatoglíficas, somatotípicas, psicológicas e fisiológicas da seleção brasileira feminina adulta de handebol. Fit. \& Perf. Jou 2006;5(2);81-86.

49. Silva ID, Dantas $P$, Mattos M. A correlação entre dermatoglifia e maturação e a sua utilização na educação física escolar. In: IX Encontro Fluminense de Educação Física Escolar. 2005: A licenciatura em foco: A formação do professor de Educação Física escolar; 2005. p. 18-22.

50. Fernandes Filho J, Alonso LV, Fernandes PR, Arêdes S, Dantas PMS. Maturação e Marcadores Genéticos. Foz do Iguaçu: The Fiep Bulletin; 2005.

Como citar: ZEQUINÃO, Marcela Almeida et al. Perfil dermatoglífico e bullying escolar em crianças e adolescentes. Cinergis, Santa Cruz do Sul, v. 18, n. 3, maio 2017. ISSN 2177-4005. Disponivel em: < https://online.unisc.br/seer/index.php/cinergis/article/ view/9007>. Acesso em: 22 jun. 2017. doi:http://dx.doi.org/10.17058/cinergis.v18i3.9007. 